\title{
Critérios e estratégias para garantir o rigor na pesquisa qualitativa
}

\section{RESUMO}

Herivelto Moreira herivelto.moreira51@gmail.com 0000-0002-2687-5089 Universidade Tecnológica Federal do Paraná - Campus Curitiba

\begin{abstract}
O objetivo do artigo é discutir a questão perene dos critérios e estratégias para assegurar maior rigor nas pesquisas qualitativas. $\mathrm{O}$ artigo foi elaborado a partir do levantamento bibliográfico em livros e artigos publicados em revistas nacionais e internacionais sobre o tema e da experiência do autor na condução e orientação de pesquisas neste paradigma. Discute a partir da natureza da pesquisa qualitativa que o respeito aos pressupostos embutidos neste paradigma no que diz respeito ao rigor merece séria consideração e, fundamentalmente, é responsabilidade dos pesquisadores que utilizam esta abordagem assegurar que isso aconteça. Além disso, analisa critérios como credibilidade, transferibilidade, dependibilidade e confirmabilidade e suas respectivas estratégias, considerando que estas questões não se constituem em fenômenos novos, pois já são discutidas há muitos anos na literatura de pesquisa. Conclui sugerindo que adotar ou não critérios ou, então, quais critérios adotar para garantir o rigor na pesquisa qualitativa é uma questão que deve ser continuamente discutida entre pesquisadores, levando em consideração as posições ontológicas, epistemológicas e as questões metodológicas práticas de cada tradição da pesquisa qualitativa.
\end{abstract}

PALAVRAS-CHAVE: Pesquisa qualitativa. Rigor. Credibilidade. Transferibilidade. Dependibilidade. Confirmabilidade. 


\section{INTRODUÇÃO}

Pesquisadores experientes de diferentes áreas do conhecimento podem muitas vezes ter dificuldades na definição de critérios que possam garantir o rigor na pesquisa qualitativa, uma vez que o rigor não está relacionado apenas ao paradigma e à intenção de pesquisa, mas também às crenças epistemológicas dos pesquisadores e a área disciplinar à qual pertencem (NORTHCOTE,2012, p.101). Portanto, não é nenhuma surpresa que pesquisadores neófitos oriundos de programas de mestrado e doutorado também tenham dificuldades para decidir se devem ou não adotar critérios e, principalmente, quais critérios adotar nas pesquisas que produzem.

Segundo Seale (1999, p.485), as discussões explícitas sobre o rigor na pesquisa social começaram a partir de preocupações com critérios de validade, confiabilidade e generalização, desenvolvidos na tradição quantitativa ou científica e se transferiu sob pressão da crítica para a tradição da pesquisa qualitativa.

Nos estudos quantitativos estes critérios estão bem estabelecidos e são amplamente aceitos como fundamentais para garantir o rigor da pesquisa (FLICK, 2009; GOLAFSHANI, 2003; KIRK e MILLER, 1986). No entanto, esses critérios são baseados em suposições sobre o pesquisador como um observador imparcial e objetivo e essencialmente relacionados à mensuração. A princípio, isso levou os pesquisadores qualitativos a criar novos termos que, ou substituíssem a linguagem científica de períodos anteriores, ou adicionassem novas ideias a essa linguagem. Neste sentido, vários pesquisadores (ARY, JACOBS e SORENSEN, 2010; CRESWELL, 1998; DENZIN e LINCOLN, 1994; GUBA, 1981; LINCOLN e GUBA, 1985) não pouparam esforços ao longo dos anos, na tentativa de fornecer orientações para julgar e assegurar o rigor nas pesquisas qualitativas, pois a ideia de fornecer um relato crível, rico e denso de como a pesquisa foi conduzida irá de alguma maneira proporcionar a outros pesquisadores ideias de como conduzir seus estudos.

O objetivo do presente artigo é discutir a questão perene dos critérios e estratégias para assegurar o rigor nas pesquisas qualitativas. $\mathrm{O}$ artigo foi elaborado a partir do levantamento bibliográfico em livros e artigos publicados em revistas nacionais e internacionais que abordam o tema e da experiência do autor na condução e orientação de pesquisas neste paradigma.

\section{A NATUREZA DA PESQUISA QUALITATIVA}

O termo pesquisa qualitativa é um termo genérico para designar um conjunto de tradições tais como a etnografia, a fenomenologia, a biografia, a teoria fundamentada, os estudos de caso, etc. Estas tradições de pesquisa utilizam diferentes perspectivas teórico-metodológicas, mas possuem características comuns e típicas da pesquisa qualitativa (MOREIRA e CALEFFE, 2008, p.60).

A pesquisa qualitativa utiliza uma abordagem naturalista que procura "compreender os fenômenos em cenários específicos, como o cenário do mundo real [onde] o pesquisador não tenta manipular o fenômeno de interesse" (PATTON, 2002, p.39). Segundo Strauss e Corbin (1990, citado por GOLAFSHANI, 2003, p.600), é possível defini-la, em termos gerais, como qualquer tipo de pesquisa que produz resultados sem a ajuda de procedimentos estatísticos ou de outros meios de quantificação, muito embora a quantificação possa ser usada. 
Apesar da importância que esta abordagem alcançou na sociedade e na academia, não faltaram críticas no que concerne aos critérios clássicos de cientificidade. A principal crítica é que ela supostamente não tem o rigor científico e credibilidade quando comparada aos métodos tradicionais quantitativos (GROULX, 2008). Por esse motivo, a pesquisa qualitativa é muitas vezes criticada como tendenciosa, de pequena escala e sem rigor científico. Na opinião de Gatti (2000, citada por ANDRÉ, 2001, p.61) essas críticas normalmente procedem, quando os resultados das pesquisas são baseados em observações casuísticas, descrições do óbvio, análises de conteúdos realizadas sem clareza metodológica, incapacidade de reflexão crítica dos vieses situacionais e, principalmente, sem a devida atenção aos critérios necessários para demonstrar a qualidade geral da pesquisa.

Neste sentido, é de suma importância que os pesquisadores qualitativos entendam os pressupostos filosóficos subjacentes a esta perspectiva paradigmática para utilizar critérios coerentes com estes pressupostos que assegurem a qualidade e o rigor nas pesquisas. As premissas que definem os paradigmas de pesquisa podem ser resumidas pelas respostas dadas pelos proponentes de qualquer paradigma às questões ontológicas, epistemológicas, metodológicas e axiológicas. Estas questões são fundamentais e, são de tal maneira inter-relacionadas, que a resposta dada a uma questão, em qualquer ordem, determina as respostas as outras questões (MOREIRA e CALEFFE, 2008, p.42).

Para os mesmos autores, a questão ontológica diz respeito à natureza ou à essência do fenômeno social investigado. Na pesquisa qualitativa acredita-se em múltiplas realidades, socialmente construídas, que geram diferentes significados para diferentes indivíduos e cuja interpretação depende do olhar do investigador. A questão epistemológica refere-se às bases do conhecimento - sua natureza e formas, como ele pode ser adquirido e como pode ser comunicado a outros seres humanos. Nesta abordagem de pesquisa a visão do conhecimento como pessoal, subjetivo e único, impõe ao pesquisador envolvimento com os participantes da pesquisa. Estas duas entidades (pesquisador e participante) estão dependentes uma da outra, sendo que a relação privilegiada do pesquisador com os participantes beneficia a pesquisa. A questão metodológica diz respeito à metodologia que o pesquisador irá utilizar, mesmo porque ontologias e epistemologias contrastantes demandarão diferentes métodos de pesquisa. $\mathrm{Na}$ pesquisa qualitativa, predominantemente utiliza-se técnicas como entrevistas, histórias de vida e observações (MOREIRA e CALEFFE, 2008, p.42-43).

$\mathrm{Na}$ questão axiológica, ou seja, o papel dos valores na pesquisa, os pesquisadores reconhecem que a pesquisa é carregada de valores e que a tendenciosidade está presente, porque o pesquisador é influenciado por tradições, ambientes e personalidades. Reconhece sua subjetividade e considera o conhecimento resultante válido para um tempo e um contexto em particular. 0 pesquisador discute abertamente os valores que determinam à narrativa e inclui a própria interpretação em conexão com a interpretação dos participantes (CRESWELL e PLANO CLARK, 2011; GUBA e LINCOLN, 1994).

Segundo vários autores (CRESWELL, 1998; DENZIN e LINCOLN, 1994; SEALE, 1999), embora os termos validade e fidedignidade e generalização sejam mais comumente associados ao rigor na pesquisa quantitativa, fazer inferências válidas 
e demonstrar a consistência dos dados coletados também são questões importantes para a pesquisa qualitativa.

\section{CRITÉRIOS E ESTRATÉGIAS PARA ASSEGURAR O RIGOR NA PESQUISA QUALITATIVA}

Toda a pesquisa é conduzida tendo como base os paradigmas existentes nas diversas áreas do conhecimento e independente do método utilizado para coletar e analisar os dados deve ter a qualidade orientada e julgada por critérios definidos. Não levar em consideração o valor dos estudos, a consistência dos métodos, o rigor na condução do estudo, a precisão dos resultados e a integridade das suposições ou conclusões obtidas poderá trazer sérias consequências, como conclusões ambíguas ou sem sentido e resultados equivocados que podem produzir um conhecimento, cuja validade científica, por vezes, é reconhecida como frágil e incerta (GROULX, 2008).

No entanto, devido à complexa e evolutiva natureza da pesquisa qualitativa, os critérios para julgar a qualidade de uma pesquisa qualitativa não estão bem resolvidos (CRESWELL, 1998) e é tema de debate contínuo (LINCOLN e GUBA, 2000). Por essas razões, é importante que os pesquisadores envolvidos com esta abordagem discutam e reflitam continuamente com seus pares e alunos os critérios considerados apropriados a este paradigma. Wolcott (1990, citado por JANESICK, 1994, p. 216), argumenta que o termo validade, que é muito valorizado no paradigma quantitativo, tornou-se confuso à medida que foi transferido para o paradigma qualitativo. A validade na pesquisa quantitativa tem um conjunto de micro definições e técnicas ao qual o leitor deste tipo de estudo está mais acostumado. Já na pesquisa qualitativa a validade tem relação com a descrição e a explicação e se a explicação se encaixa ou não na descrição. Em outras palavras, se a explicação é crível. Sendo assim, os termos validade, fidedignidade e generalização, da maneira como são utilizados no paradigma quantitativo são muitas vezes considerados inadequados para julgar o rigor da pesquisa qualitativa, mesmo porque eles não foram concebidos para esse fim (DENZIN e LINCOLN, 1994; GUBA e LINCOLN, 1995; MERTENS, 2005).

O rigor na pesquisa qualitativa é definido por um conjunto oposto de critérios utilizados na pesquisa quantitativa e está associado ao pesquisador estar aberto aos dados, aderir meticulosamente a uma perspectiva filosófica específica e ao rigor na coleta e na análise dos dados. Na opinião de Macedo, Galeffi e Pimentel, pensar rigorosamente o rigor na pesquisa qualitativa é compreender sua contrapartida complementar: a flexibilidade. Rigor e flexibilidade andam juntos na pesquisa qualitativa, porque o excesso de rigidez deve ser corrigido ou equilibrado com a flexibilidade, assim como o excesso de flexibilidade tem que ser corrigido com o tensionamento justo (MACEDO, GALEFFI e PIMENTEL, 2009, p.38).

A partir do argumento dos autores acima, é recomendável utilizar alguns critérios para assegurar o rigor e a extensão pela qual os resultados da pesquisa qualitativa são apoiados em evidências, mas com equilíbrio entre o rigor e a flexibilidade, pois sem a adoção de critérios adequados e sem o controle teóricometodológico permanente do próprio processo de interpretação, os estudos qualitativos dificilmente escaparão as críticas atribuídas a esse tipo de pesquisa. 
No entanto, não existe uma posição única entre os pesquisadores que adotam a abordagem qualitativa em relação a usar ou não critérios e quais critérios usar, mas sim uma variedade de posições que diferem de acordo com diferentes tradições da pesquisa qualitativa (FLICK, 2009). No entanto, está fora do escopo deste artigo discutir cada uma destas posições dentro das várias tradições. De maneira geral, estas posições variam de ignorar qualquer tentativa de estabelecer rigor utilizando os conceitos de validade, fidedignidade e generalização inerentes ao paradigma quantitativo, até usar termos e critérios que levam em consideração as especificidades da pesquisa qualitativa. Esta última posição é bem-vinda e será tratada na sequência do artigo.

Estimulados pelo trabalho pioneiro de Guba (1981) sobre os critérios emergentes para assegurar maior rigor na pesquisa qualitativa, vários pesquisadores adotaram e também sugeriram critérios. Dentre os vários critérios discutidos e sugeridos nas várias tradições da pesquisa qualitativa, adotamos para este artigo os critérios de credibilidade, transferibilidade, dependibilidade e confirmabilidade (DENZIN e LINCOLN, 1994; GUBA, 1981; VIDICH e LYMAN, 1994).

\section{CREDIBILIDADE}

Para Guba e Lincoln (1989), a credibilidade na pesquisa qualitativa diz respeito à veracidade dos resultados da pesquisa e envolve o quão bem o pesquisador estabeleceu a confiança nos resultados baseado no delineamento do estudo, nos participantes e no contexto. A credibilidade estabelece ou não se os resultados da pesquisa representam informações críveis extraídas dos dados originais e se é uma interpretação correta do ponto de vista dos participantes. Neste sentido, Ary, Jacobs e Sorensen (2010, p.498), enfatizam que o pesquisador tem o compromisso de representar as realidades dos participantes da pesquisa e proporcionar garantias deste compromisso no relatório de pesquisa. Sendo assim, uma descrição detalhada do processo e dos resultados da pesquisa fornece aos leitores base para julgar a credibilidade dos estudos. Esta descrição densa contribuirá para determinar o nível de confiança nos resultados relatados, pois oportuniza também aos leitores verificar como foram realizados todos os procedimentos específicos para a coleta e a análise dos dados. Para Maykut e Morehouse (1995, p.145) o termo credibilidade na pesquisa qualitativa é análogo à validade interna na pesquisa quantitativa.

Várias estratégias foram identificadas na literatura para dar credibilidade aos estudos qualitativos. Denzin e Lincoln (1994, p.513) sugerem as seguintes estratégias: a triangulação, a revisão por pares (peer reviews), a verificação pelos participantes (member check), o trabalho de campo estendido, a reflexividade e a amostragem de caso negativo. Cada uma destas estratégias é discutida em detalhes nas subsecções abaixo.

\section{TRIANGULAÇÃO}

O principal defensor do método da triangulação é Norman Denzin (1970). Para o autor, o principal objetivo da triangulação é contornar as tendenciosidades pessoais dos pesquisadores para superar as deficiências inerentes a apenas um investigador, uma teoria única ou um único método, aumentando assim a 
credibilidade do estudo. Existem três técnicas de triangulação (DENZIN, 1970; DENZIN e LINCOLN, 2005; LINCOLN e GUBA, 1985).

A primeira técnica é a triangulação de métodos/dados que utiliza diferentes métodos tais como entrevistas, grupo focal ou observação participante, fontes de dados ou ainda diferentes informantes para melhorar a qualidade dos dados. No entanto, Denzin (1970) faz uma distinção entre a triangulação dentro dos métodos e a triangulação entre os métodos.

Para Denzin (1970, citado por MOREIRA e CALEFFE, 2008, p.192), a triangulação dentro dos métodos refere-se à replicação de um estudo, usando as mesmas técnicas como forma de verificar a credibilidade de um estudo e a natureza das teorias geradas. A triangulação entre os métodos refere-se ao uso de mais de um método para coletar dados em um único estudo. Dada às circunstâncias, o pesquisador provavelmente utilizará uma ou outra triangulação metodológica como método para verificar a credibilidade dos dados coletados.

A segunda técnica é a triangulação de pesquisadores para investigar o mesmo problema e trazer diferentes percepções do fenômeno para ajudar a fortalecer a integridade dos resultados. Normalmente, isso ocorre por meio de uma equipe de pesquisa constituída por colegas em um campo de estudo em que cada investigador examina o mesmo estudo a partir de sua perspectiva. A terceira é a triangulação teórica que envolve o uso de múltiplas perspectivas para interpretar um único conjunto de dados. Denzin (1970) também argumenta que ao contrário da triangulação de pesquisadores, esta estratégia normalmente implica na utilização de profissionais de outras áreas do conhecimento. Uma abordagem popular é reunir pessoas de diferentes áreas disciplinares. No entanto, indivíduos dentro das mesmas áreas disciplinares podem ser utilizados, desde que eles tenham diferentes posições em relação ao assunto.

\section{REVISÃO POR PARES}

A revisão por pares requer ter um pesquisador externo qualificado para examinar os processos de pesquisa e as interpretações dos dados. O revisor verifica se os dados foram coletados e analisados de forma adequada e sistemática e, em muitos casos, que conclusões foram retiradas (GLESNE e PESHKIN, 1992; MILES e HUBERMAN, 1994).

Nesta estratégia, o pesquisador explora a análise e as conclusões do pesquisador externo em bases contínuas. Nas dissertações e teses, os orientadores podem desempenhar este papel fundamental com os alunos para garantir que isso aconteça (MERTENS, 2005).

A revisão por pares pode ser obtida de inúmeras formas. Uma delas é discutir os resultados emergentes com colegas da mesma área do conhecimento; uma segunda é apresentar e defender o método e as conclusões em congressos nacionais e internacionais a outros investigadores para atrair o comentário crítico e, uma terceira, é apresentar os resultados e as implicações para grupos interessados sobre o assunto.

Na opinião de Long e Johnson (2000, p.34), a revisão por pares destina-se a estimular a consideração e a exploração de perspectivas e explicações adicionais 
em várias fases do processo de coleta e análise de dados. Em particular, visa prevenir o encerramento prematuro da busca de sentido e de padrões nos dados.

\section{VERIFICAÇÃO PELOS PARTICIPANTES}

A verificação pelos participantes é definida como um processo pelo qual o pesquisador procura melhorar a precisão e a credibilidade do que foi gravado, por exemplo, durante uma entrevista (GLESNE e PESHKIN, 1992; LINCOLN e GUBA, 1985; MAYKUTE e MOREHOUSE, 1994; MILES e HUBERMAN, 1994).

Em geral, durante a entrevista, o pesquisador irá reformular ou resumir as informações e, em seguida, apresentá-las aos participantes para que eles determinem a precisão. $O$ estudo adquire credibilidade quando os participantes concordam que os resumos refletem suas opiniões, sentimentos e experiências e se a exatidão e a integridade são confirmadas (CRESWELL 2007; LINCOLN e GUBA, 1985).

Outro tipo de verificação pelos participantes pode ocorrer próximo ao final da pesquisa quando os dados analisados e o relatório são enviados aos participantes para avaliar sua autenticidade. Os participantes verificam se foi feita a representação verdadeira ou autêntica do que falaram durante as entrevistas. Isso pode envolver o compartilhamento de todos os resultados com os participantes, permitindo-Ihes analisar criticamente os resultados e as interpretações sobre eles (CRESWELL, 2007; LINCOLN e GUBA, 1985).

Se a verificação pelos participantes ocorrer simultaneamente durante a entrevista ou próxima do final da pesquisa, estas verificações não são irrepreensíveis, mas servem para diminuir a incidência de dados e de informações incorretas, de forma a fornecer resultados que sejam autênticos e originais (CRESWELL, 2007; MOUSTAKAS, 1994).

Mertens (2005, p.255) argumenta que a verificação pelos participantes é uma das estratégias mais importantes para dar credibilidade aos estudos qualitativos e reforça que ela pode ser formal ou informal. Por exemplo, ao final de uma entrevista, o pesquisador pode resumir o que foi dito e perguntar se as anotações refletem precisamente a posição da pessoa. Os rascunhos do relato da pesquisa podem ser compartilhados com os participantes para que eles leiam e comentem.

O maior benefício desta estratégia é que ela oportuniza ao pesquisador verificar a exatidão e a integridade dos resultados que na sequência poderá ajudar a melhorar a credibilidade do estudo. Os pesquisadores não devem temer que os dados sejam contaminados pelo participante, pois a boa pesquisa deve retornar aos participantes para que então, o pesquisador possa aperfeiçoar os resultados à luz das suas reações.

Esta estratégia é particularmente valiosa na pesquisa-ação, onde os pesquisadores trabalham com os participantes em uma base contínua para facilitar a mudança. A maioria das pesquisas, no entanto, envolve a coleta de dados com entrevistas individuais e observações de pequenas amostras em que a verificação pelos participantes pode, muitas vezes, gerar mais problemas do que contribuições. 


\section{TRABALHO DE CAMPO ESTENDIDO}

Para ajudar tanto a descoberta, quanto a validação dos dados, os pesquisadores deveriam coletar dados no campo durante um período de tempo prolongado. Kirk e Miller (1986) argumentam que o envolvimento prolongado no cenário da pesquisa aumenta a sensibilidade do pesquisador para as discrepâncias entre os significados presumidos pelo investigador e os significados atribuídos pelos participantes ao fenômeno que está sendo investigado.

Guba e Lincoln $(1989$, p. 237) recomendam esta estratégia para construir a confiança e superar as dificuldades apresentadas por construções perversas e pela desinformação por parte dos entrevistados. Segundo Mertens (2005, p.254), a observação persistente aumenta o efeito desse envolvimento por permitir que o pesquisador "identifique essas características e elementos da situação que são mais relevantes ao problema para então focá-las com mais detalhes"

No entanto, não há uma regra rígida que diga quanto tempo o pesquisador deve permanecer no campo. Quando o pesquisador sente que os temas, informações e exemplos estão se repetindo ao invés de se estender, é a hora de abandonar a coleta de dados. Isto é o que Bogdan e Biklen $(1994$, p.96) denominam de saturação de dados, ou seja, o ponto da coleta de dados a partir da qual a aquisição de informação se torna redundante.

\section{TENDENCIOSIDADE DO PESQUISADOR}

Ary, Jacobs e Sorensen (2010, p.500) argumentam que a tendenciosidade do pesquisador pode ser prejudicial na pesquisa qualitativa, pois pode resultar em observações seletivas, ouvir apenas aquilo que se quer ouvir, ou permitir que atitudes, preferências e sentimentos afetem a interpretação dos dados. Para tentar evitar que isto aconteça, Creswell (1998) enfatiza que o pesquisador deve esclarecer a sua posição desde o início do estudo. Isto é importante para que o leitor entenda a posição do pesquisador e qualquer tendenciosidade ou suposição que possa influenciar a investigação. Outras estratégias para controlar a tendenciosidade do pesquisador em estudos qualitativos são a reflexividade e a amostragem de caso negativo, estratégias que serão discutidas a seguir.

\section{REFLEXIVIDADE}

A reflexividade envolve a reflexão crítica de como o pesquisador constrói o conhecimento a partir do processo de investigação, dos tipos de fatores que influenciam a construção do conhecimento do pesquisador e como essas influências são reveladas no planejamento, na condução e no relatório da pesquisa. Para GUILLEMIN e GILLAM, o pesquisador reflexivo é aquele que tem conhecimento de todas essas influências potenciais e é capaz de voltar atrás e olhar criticamente o seu próprio papel no processo de investigação. $O$ objetivo de ser reflexivo nesse sentido tem a ver com a melhoria da qualidade e da validade da pesquisa e reconhecer as limitações do conhecimento que é produzido, levando assim a uma investigação mais rigorosa (GUILLEMIN e GILLAM, 2004, p. 276). subjacente à precisão dos resultados da pesquisa qualitativa - ou seja, o 
componente da interação social da relação entrevistador-entrevistado, ou seja, as relações de poder, muitas vezes assimétricas, que se estabelecem entre o pesquisador e o participante do estudo.

Para Creswell (1998) a reflexividade significa que o pesquisador é consciente de sua tendenciosidade, valores e experiências que são trazidas para a pesquisa qualitativa e, sendo assim, devem estar explícitas no texto.

\section{AMOSTRAGEM DE CASO NEGATIVO}

A amostragem de caso negativo (ARY, JACOBS e SORENSEN, 2010; CRESWELL, 1998; GUBA e LINCOLN, 1989; LINCOLN e GUBA, 1985; MERTENS, 2005; MILES e HUBERMAN, 1994) diz respeito à localização e o exame de casos que refutam as expectativas do pesquisador. Isso envolve procurar e discutir elementos nos dados que não dão suporte ou parecem contradizer padrões ou explicações que estão surgindo a partir da análise dos dados. Esta estratégia, se constitui em um processo para aperfeiçoar a análise até que o pesquisador possa explicar ou responder pela maioria dos casos que tem por finalidade ampliar e confirmar os padrões emergentes da análise dos dados.

Um exemplo de análise de caso negativo é o pesquisador aperfeiçoar uma hipótese até que ela aborde todos os casos nos dados. Se o estudo inclui a produção de tipologias, ao completar as categorias iniciais o investigador pode rever os dados para confirmar se essas construções, de fato, são responsáveis por todas as ocorrências do fenômeno envolvido, mesmo que alguns dos tipos se refiram apenas a uma ocorrência (LINCOLN e GUBA, 1985; MILES e HUBERMAN, 1994).

\section{TRANSFERIBILIDADE}

É o grau pelo qual os resultados de um estudo qualitativo podem ser aplicados ou generalizados para outros contextos ou para outros grupos (DENZIN e LINCOLN, 1994). Embora os pesquisadores que adotam o paradigma qualitativo não tenham a generalização como meta, é de sua responsabilidade fornecer descrições suficientemente ricas, detalhadas e densas do contexto, de maneira que os potenciais usuários possam fazer as necessárias comparações e julgamentos sobre a similaridade e, consequentemente, sobre a transferibilidade dos resultados. Isso é chamado de adequação descritiva (ARY, JACOBS e SORENSEN, 2010).

As descrições extensas e cuidadosas do tempo, local, contexto, e cultura são obtidas por meio da "descrição rica e densa". A descrição rica e densa permite ao leitor tomar decisões em relação à transferibilidade (LINCOLN e GUBA, 1985) porque o pesquisador descreve em detalhes os participantes e o cenário do estudo. Para Erlandson et al. (1993, citados por PICKERT e DIXON, 2004), com tal descrição detalhada, os pesquisadores oportunizam aos leitores transferir informações para outros cenários e determinar se os resultados mostram características compartilhadas.

Neste sentido, o pesquisador precisa empenhar-se para fornecer descrições precisas detalhadas e completas do contexto e dos participantes para ajudar o leitor a determinar a transferibilidade. Alguns pesquisadores qualitativos 
argumentam que é possível aplicar resultados qualitativos para outras pessoas, cenários e tempos na medida em que eles sejam similares às pessoas, cenários e tempos do estudo original. Isso contrasta com a generalização na pesquisa quantitativa onde o pesquisador é quem faz a generalização.

Para Mertens (2005), o fardo da transferibilidade na pesquisa qualitativa está no leitor para determinar o grau de similaridade entre o local do estudo e o contexto receptor. A responsabilidade do pesquisador é fornecer detalhes suficientes que possibilitem ao leitor fazer tal julgamento. Outra estratégia para proporcionar a transferibilidade é incluir a comparação entre casos. $O$ pesquisador pode investigar mais de um caso e, se os resultados forem similares, aumenta a possibilidade de transferibilidade dos resultados para outros cenários.

O pesquisador deve estar atento de que existem ameaças a transferibilidade tais como, efeitos da seleção (o fato de que os fenômenos que estão sendo investigados são únicos para determinado grupo), efeitos do contexto (o fato de que os resultados podem ser função do contexto específico da investigação), e efeitos da história (o fato de que experiências históricas únicas dos participantes possam se opor as comparações). $O$ pesquisador deve reconhecer as limitações do estudo na descrição. Detalhar as circunstâncias ajuda o leitor entender a natureza dos dados e o que pode ser peculiar para o estudo.

Segundo Ary, Jacobs e Sorensen (2010, p.502), a reatividade, isto é, o efeito do pesquisador na pesquisa se refere ao potencial do pesquisador ou dos procedimentos para influenciar os participantes, mudando assim os resultados do estudo. A reatividade também pode limitar a transferibilidade. Os mesmos autores ainda enfatizam que embora eliminar a influência do pesquisador possa ser impossível em um estudo qualitativo, porque o pesquisador é o instrumento chave na coleta de dados, o pesquisador pode ajudar o leitor a entender a sua influência descrevendo suas próprias tendenciosidades por meio de uma declaração reflexiva e fornecendo descrições detalhadas das estratégias de observação e perguntas da entrevista. A reatividade é séria ameaça em estudos que usam técnicas de entrevistas (ARY, JACOBS e SORENSEN (2010, p.501).

\section{DEPENDIBILIDADE}

Vários pesquisadores (ARY, JACOBS e SORENSEN, 2010; GUBA e LINCOLN, 1994; DENZIN, 1994; MERTENS, 2005; VIDICH e LYMAN, 1994; entre outros) usam o termo dependibilidade ao invés de fidedignidade. A verificação da dependibilidade pode ser conduzida para atestar a qualidade e a adequação do processo de pesquisa. Lembremos que a fidedignidade na pesquisa quantitativa tem a ver com consistência do comportamento, ou a medida pela qual os dados e os resultados seriam similares se o estudo fosse replicado. Assim, a dependibilidade é vista como a medida pela qual a variação pode ser localizada ou explicada. Algumas estratégias para investigar a dependibilidade são: o exame da trilha (audit trail), a replicação lógica, a replicação gradativa.

\section{EXAME DA TRILHA}


(MAYKUT e MOREHOUSE, 1995, p.146). Esta estratégia documenta como o estudo foi conduzido, incluindo o que foi feito, quando e por que. $O$ exame da trilha contém os dados crus obtidos nas entrevistas e nas observações das decisões dos investigadores sobre quem entrevistar ou o que observar e por que. Estes dados podem ser gravados em arquivos para documentar como o problema e os objetivos da pesquisa foram desenvolvidos e, subsequentemente, aprimorados.

O pesquisador deve manter anotações minuciosas e gravações das atividades e manter os dados bem organizados e de uma maneira recuperável. Deve fornecer informações sobre os participantes, o processo de seleção amostral, descrições contextuais, métodos de coleta dos dados, anotações de campo detalhadas, métodos de análise dos dados, gravações em áudio, filmes e outros materiais descritivos que possam ser revistos por outras pessoas.

Ao usar o exame da trilha como guia, um segundo ou terceiro pesquisador independente pode examinar o estudo para atestar a dependibilidade dos procedimentos empregados e examinar se os resultados são confirmáveis, isto é, se eles são logicamente derivados de, e fundamentados nos dados que foram coletados. Uma apresentação completa dos procedimentos e dos resultados possibilita que o leitor faça julgamentos sobre a replicabilidade da pesquisa dentro dos limites do contexto original (LONG e JOHNSON, 2000; MAYKUT e MOREHOUSE, 1995).

\section{REPLICAÇÃO LÓGICA}

A replicação lógica envolve a condução de um estudo em múltiplos locais ou com múltiplos grupos. Esta estratégia é sugerida por Ary, Jacobs e Sorensen (2010) para determinar a dependibilidade de um estudo. A dependibilidade também pode ser demonstrada mostrando a consistência dos resultados através de múltiplos contextos ou múltiplos investigadores. De acordo com esta lógica, quanto mais vezes um resultado é encontrado e é verdadeiro em diferentes conjuntos de pessoas ou em diferentes contextos e períodos de tempo, mais confiante o pesquisador pode estar nas suas conclusões.

\section{REPLICAÇÃO GRADATIVA}

A replicação gradativa é outra estratégia sugerida por Ary, Jacobs e Sorensen (2010) para determinar a dependibilidade. Nesta estratégia, dois investigadores dividem os dados, os analisam independentemente e comparam os resultados. A consistência dos resultados fornece evidências da dependibilidade. 0 exemplo mais comum é quando duas equipes de investigação lidam individualmente com os dados que tenham sido divididos e os resultados são contrastados. Um aspecto importante na replicação gradativa é que a comunicação entre as equipes e os membros da equipe é vital. Deve haver uma disposição para a comunicação em base regular e em pontos pré-definidos no processo de pesquisa.

Ainda em relação à dependibilidade, o pesquisador deve demonstrar que os métodos usados são reproduzíveis e consistentes, que a abordagem e os documentados e que a evidência externa pode ser usada para testar as conclusões. 
A concordância entre avaliadores em relação aos resultados também é uma estratégia para determinar a dependibilidade. Um dos métodos que pode ser utilizado é o de codificar-recodificar: um pesquisador codifica os dados, abandona a análise por um período de tempo e, então, retorna e recodifica os dados e os compara com a codificação obtida pelo outro avaliador.

Devido a algumas pesquisas qualitativas envolverem a observação por diferentes observadores, alguns pesquisadores sugerem métodos interobservadores de avaliação da dependibilidade. Por exemplo, um pesquisador poderia selecionar aleatoriamente uma transcrição e solicitar a um colega que codifique a transcrição usando os rótulos de códigos identificados pelo pesquisador. $\mathrm{O}$ segundo codificador poderá livremente adicionar outros códigos que identificar. Após o colega completar a codificação das transcrições, os resultados são comparados à codificação original para determinar se ambos os codificadores, codificaram as transcrições da mesma maneira. A triangulação, já discutida anteriormente, também pode ser usada para estabelecer a dependibilidade dos estudos qualitativos.

\section{CONFIRMABILIDADE}

A confirmabilidade na pesquisa qualitativa tem o mesmo conceito de objetividade na pesquisa quantitativa. Ambos lidam com a ideia de neutralidade ou a medida pela qual o pesquisador é livre de tendenciosidades nos procedimentos e na interpretação dos resultados (ARY, JACOBS e SORENSEN, 2010, p.504).

Mertens (2005, p.256) aprofunda um pouco mais esta questão argumentando que a confirmabilidade significa que os dados e sua interpretação não são invenções da imaginação do pesquisador, pois os dados qualitativos devem ser rastreados até sua fonte e a lógica que foi usada para interpretar os dados deverá ser explicitada.

Devido à impossibilidade de atingir os níveis de objetividade que os estudos quantitativos se esforçam para obter, os pesquisadores qualitativos não estão preocupados se os dados que coletam e as conclusões que chegam serão confirmados por outros que investiguem a mesma situação. Assim, em estudos qualitativos, o foco muda da neutralidade do pesquisador para a confirmabilidade dos dados e das interpretações.

Segundo Maykut e Morehouse (1995, p.146) o exame da trilha é a principal estratégia para demonstrar a confirmabilidade. Ao fornecer o exame da trilha o pesquisador oportuniza que outros pesquisadores cheguem ou não aos mesmos dados, conclusões e contextos. Outras estratégias usadas para determinar a confirmabilidade podem incluir a triangulação de métodos, a revisão por pares e a reflexividade, já discutidos anteriormente.

Guba e Lincoln (1989) recomendam a verificação da confirmabilidade para atestar o fato de que os dados podem ser rastreados às fontes originais e de que o processo para sintetizar os dados para chegar à conclusão possa ser confirmado. A verificação da confirmabilidade poderá ser conduzida em conjunto com a verificação da dependibilidade. Assim, outros pesquisadores poderão revisar as notas de campo, transcrições de entrevistas e determinar se as conclusões foram apoiadas nos dados. 
As questões fundamentais para assegurar maior rigor nas pesquisas qualitativas dizem respeito a se o pesquisador: mostrou a coesão das evidências, registrou os dados com precisão e elaborou os códigos de maneira uniforme e consistente, capturou com precisão a compreensão social ou a realidade social subjacente aos eventos, atividades e comportamentos, mostrou a adequação das evidências e proporcionou a descrição densa do fenômeno que está sendo estudado. A Tabela 1 mostra de maneira resumida os critérios para assegurar o rigor e as possíveis estratégias e ações do pesquisador.

Critérios para assegurar o rigor

\section{Possíveis estratégias e ações do pesquisador}

Adoção de métodos de pesquisa adequados ao problema e reconhecidos. Desenvolvimento de familiaridade inicial com a cultura dos participantes e das organizações.

Descrição dos antecedentes e da experiência do pesquisador.

Triangulação de diferentes métodos, tipos de informantes, pesquisadores e teorias. Revisão pelos pares - verificação dos dados coletados e interpretações/teorias

Credibilidade formadas pelos pares. Verificação pelos respondentes. Trabalho de campo estendido permanecer no campo o tempo necessário, principalmente em estudos de caráter etnográfico e pesquisa-ação.

Reflexividade - comentário reflexivo. Amostragem de caso negativo. Ações para ajudar a garantir a honestidade dos participantes.

Questionamento iterativo dos diálogos na coleta de dados.

Sessões de esclarecimento entre pesquisador e orientador.

Descrição densa do fenômeno e do contexto a ser estudado para permitir comparações.

Fornecer dados dos antecedentes para estabelecer o contexto do estudo e

Transferibilidade descrição detalhada do fenômeno em questão.

Escrever as tendenciosidades do pesquisador por meio de uma declaração reflexiva, fornecendo descrições detalhadas das estratégias de observação e perguntas da entrevista.

Exame da trilha - documentar como o estudo foi conduzido, incluindo o que foi feito, quando e por que.

Dependibilidade Manter anotações minuciosas e gravações das atividades. uma maneira facilmente recuperável. 
Fornecer informações sobre os

participantes, o processo de seleção

amostral, descrições contextuais, métodos

de coleta dos dados, anotações de campo

detalhadas, métodos de análise dos dados, gravações em áudio, filmes e outros materiais descritivos que possam ser revistos por outros pesquisadores e leitores.

Replicação lógica - conduzir o estudo em múltiplos locais ou com múltiplos grupos. Replicação gradativa - dois pesquisadores dividem os dados, os analisam independentemente e comparam os resultados.

Exame da trilha - diagramas para

demonstrar o exame da trilha.

Triangulação de métodos para reduzir o efeito da influência do pesquisador. Revisão pelos pares.

Confirmabilidade Reflexividade.

Admissão de crenças e pressupostos do pesquisador.

Reconhecimento de falhas nos métodos e seus efeitos potenciais na descrição da metodologia para permitir a verificação da integridade dos resultados da investigação.

\section{CONSIDERAÇÕES FINAIS}

O objetivo do artigo foi abordar a questão perene dos critérios e suas estratégias para assegura o rigor nas pesquisas qualitativas. $O$ artigo não teve a pretensão de esgotar o tema ou sugerir que estes são os únicos critérios existentes e, reconhece que ao tratar esta questão adentra-se em um cenário complexo, conflituoso e nebuloso, pois existem várias tradições de pesquisa qualitativa, com reivindicações concorrentes em relação ao que conta como rigor em cada uma delas.

Neste sentido, o rigor da pesquisa deve ser julgado em termos do seu próprio paradigma, da tradição de pesquisa, do campo de investigação e da área disciplinar. Por exemplo, enquanto os termos de validade e fidedignidade e generalização são critérios essenciais para julgar o rigor na pesquisa no paradigma quantitativo, os termos credibilidade, transferibilidade, dependibilidade e confirmabilidade podem ser critérios para julgar o rigor nas pesquisas no paradigma qualitativo (LINCOLN e GUBA, 1985).

O respeito aos pressupostos embutidos no paradigma qualitativo de pesquisa merece séria consideração, principalmente por parte dos pesquisadores. É necessário buscar sempre a melhoria da qualidade dos estudos e assumir como tarefa estabelecer critérios para assegurar maior rigor na pesquisa e apresentá-los publicamente para ouvir críticas e sugestões.

Em um mundo de múltiplas realidades, múltiplas verdades podem existir e isso significa que no paradigma qualitativo os significados associados com os termos 
validade, fidedignidade e generalização são muito diferentes dos significados apresentados no paradigma quantitativo.

Na verdade, as noções de validade, fidedignidade e generalização usadas na pesquisa quantitativa podem não fazer sentido na pesquisa qualitativa, implicando alguma verdade impessoal e automática. A aplicação das concepções quantitativas de validade, fidedignidade e generalização são inadequadas para julgar o rigor na pesquisa qualitativa, pois tem o potencial de criar a impressão de que ela não é uma abordagem academicamente rigorosa, pelo menos em comparação aos métodos quantitativos. É, no entanto, igualmente inadequado supor que os critérios que são adequados para julgar o rigor na pesquisa qualitativa são inatingíveis, ou estão indisponíveis. Critérios academicamente rigorosos estão disponíveis e acessíveis para tal julgamento.

Adotar ou não critérios ou, então, quais critérios adotar para garantir o rigor na pesquisa qualitativa são questões que devem ser continuamente discutidas entre os pesquisadores, levando em consideração as posições epistemológicas e as questões metodológicas práticas de cada tradição da pesquisa qualitativa. No entanto, não é necessário que o pesquisador adote todas as estratégias. Recomenda-se aos pesquisadores adotar pelo menos duas estratégias em referência a cada critério discutido.

Neste sentido, os pesquisadores têm a responsabilidade de assegurar maior rigor em suas pesquisas, uma vez que a pesquisa quantitativa é frequentemente vista como o método preferido e aceito de investigação e, em alguns contextos, o valor da pesquisa qualitativa pode ser questionado por pesquisadores de outras orientações filosóficas. Assim, o papel do pesquisador neste processo é, muitas vezes, o de dissipar equívocos sobre a própria natureza da pesquisa qualitativa. 


\title{
Criteria and strategies to ensure rigor in qualitative research
}

\begin{abstract}
The objective of the article is to discuss the perennial question of the criteria and strategies to ensure rigor in qualitative research. The article was elaborated based on a literature review in books and articles published in national and international journals, and on the author's experience conducting and supervising research in this paradigm. Discusses from the nature of qualitative research that the respect for the assumptions built into this paradigm with regard rigor deserves serious consideration, and fundamentally, it is the responsibility of researchers using this approach to ensure that this happens. It analyzes criteria such as credibility, transferability, dependability, and confirmability and its strategies, considering that these issues are not new phenomenon and have existed for many years in the research literature. It concludes by suggesting that to adopt or not criteria or what criteria to adopt to ensure the rigor of qualitative research is an issue that must be continually discussed among researchers taking into account the epistemological positions and the practical methodological issues of each tradition in qualitative research.
\end{abstract}

KEYWORDS: Qualitative research. Rigor. Credibility. Transferability. Dependability. Confirmability. 


\section{REFERÊNCIAS}

ANDRÉ, Marli. Pesquisa em Educação: buscando rigor e qualidade. Cadernos de Pesquisa, n.113, p.51-64, julho/2001.

ARY, Donald.; JACOBS, Lucy; SORENSEN Chris. Introduction to research in education. 8th Edition. Canada: Wadsworth Cengage Learning, 2010.

BOGDAN, Robert e BIKLEN, Sari. A investigação qualitativa em educação: uma introdução à teoria e aos métodos. Porto: Porto Editora, 1994.

CRESWELL, John. Qualitative inquiry and research design: choosing among five traditions. Thousand Oaks, CA: Sage Publications, 1998.

CRESWELL, John. Qualitative inquiry and research design: Choosing among five traditions. 2nd ed. Thousand Oaks, CA: Sage, 2007.

CRESWELL, John; PLANO CLARK, Vicki. Designing and conducting mixed methods research. Sage: Thousand Oaks, California, 2011.

DENZIN, Norman. The research act. 2.ed. Chicago: Aldine, 1970.

DENZIN, Norman; LINCOLN, Yvonna. Handbook of qualitative research. Sage, London. 1994.

DENZIN, Norman. The art and politics of interpretation. In: DENZIN, Norman; LINCOLN, Yvonna. Handbook of qualitative research. Sage, London. 1994, p.502515.

DENZIN, Norman; LINCOLN, Yvonna. (Eds.). The sage handbook of qualitative research. Thousands Oaks, CA: Sag, 2005.

FLICK, Uwe. Introdução a pesquisa qualitativa. 3 ed. Porto Alegre: Artmed, 2009.

GLESNE, Corrine; PESHKIN, Alan. Becoming qualitative researchers: an introduction. White Plains, NY: Longman, 1992.

GOLAFSHANI, Nahid. Understanding reliability and validity in qualitative research. The Qualitative Report, v.8, n.4, p. 597-607, December 2003. 20 jul. 2016. 
GROULX, L. Contribuição da pesquisa qualitativa à pesquisa social. In: POUPART, J. et al. A pesquisa qualitativa: enfoques epistemológicos e metodológicos. Petrópolis: Vozes, 2008. p.95-125.

GUBA, Egon; LINCOLN, Yvonna. Fourth generation evaluation. Newbury Park: Sage, 1989.

GUBA, Egon. Criteria for assessing the trustworthiness of naturalistic inquiry. Education, Communication and Technology Journal, n.29, v.2, p.75-91, 1981. Disponível em: http://www.jstor.org/page/info/about/policies/terms.jsp. Acesso em: 23 jun.2016.

GUBA, Egon; LINCOLN, Yvonna. Competing paradigms in qualitative research. In: DENZIN, Norman and LINCOLN, Yvonna (Editors). Handbook of qualitative research. Thousand Oaks: Sage Publications, 1994, p.105-117.

GUBA, Egon; LINCOLN, Yvonna. Fourth generation evaluation. Sage, Newbury Park, CA, USA. 1995.

GUILLEMIN, Marilys; GILLAM, Lynn. Ethics, reflexivity, and "ethically importante moments in research. Qualitative Inquiry, v.10, n.2, p.261-280, 2004. DOI: 10.1177/1077800403262360. Disponível em: https://www.researchgate.net/publication/235930722_Ethics_Reflexivity_and_E thically Important_Moments_in_Research. Acesso em: 02 ag. 2016.

JANESICK, Valerie. The dance of qualitative research design: metaphor, methodolatry, and meaning. In: DENZIN, N. e LINCOLN, Y. (Eds.). Handbook of qualitative research. Thousand Oaks, Sage, 1994. p.209-219.

KIRK, Jerome; MILLER, Marc. Reliability and validity in qualitative research. Beverly Hills: Sage Publications, 1986.

LINCOLN, Yvonna; GUBA, Egon. Naturalistic inquiry. Beverly Hills: Sage, 1985.

LINCOLN, Yvonna; GUBA, Egon. Paradigmatic controversies, contradictions and emerging confluences. In: DENZIN, Norman; LINCOLN Yvonna. (Eds.), Handbook of qualitative research. 2nd ed. California: Sage Publications. 2000. p.163-188.

LONG, Tony; JOHNSON, Martin. Rigour, reliability and validity in qualitative research. Clinical Effectiveness in Nursing, (4), p.30-37, 2000. Disponível em: http://www.sciencedirect.com/science/article/pii/S1361900400901067. Acesso em: 11 ag. 2016 doi.org/10.1054/cein.2000.0106. 
MACEDO, Roberto Sidnei, GALEFFI, Dante; PIMENTEL, Álamo. Um rigor outro sobre a qualidade na pesquisa qualitativa: educação e ciências humanas [online]. Salvador: EDUFBA, 2009. Disponível em: SciELO Books. http://books.scielo.org. Acesso em: 20 jun. 2016.

MAYKUTE, Pamela; MOREHOUSE, Richard. Beginning qualitative research: a philosophic and practical guide. London: Falmer Press, 1995.

MERTENS, Donna. Research evaluation in education and psychology: integrating diversity with quantitative, qualitative, and mixed methods. 2 nd edition. London: Sage, 2005.

MILES, Mathew; HUBERMAN, Michael. Qualitative data analysis: an expanded sourcebook, 2nd ed. California: Sage, 1994.

MOREIRA, Herivelto e CALEFFE, Luiz Gonzaga. Metodologia da pesquisa para o professor pesquisador. Rio de Janeiro: Lamparina, 2008.

MOUSTAKAS, Clark. Phenomenological research methods. Thousand Oaks, CA.: Sage, 1994.

NORTHCOTE, Maria T. Selecting criteria to evaluate qualitative research. Education Papers and Journal Articles. Paper 38. p.98-110, 2012. Disponível em: http://research.avondale.edu.au/edu_papers/38. Acesso em: 21 jul 2016.

PATTON, Michael Quinn. Qualitative evaluation and research methods. 2nd ed. Newbury Park: Sage, 1990.

PATTON, Michael Quinn. Qualitative evaluation and research methods. 3rd ed. Thousand Oaks, CA: Sage Publications. 2002.

PICKARD, Alison; DIXON, Pat. The applicability of constructivist user studies: How can constructivist inquiry inform service providers and systems designers? Information Research, n.9, v.3, p. 2004. Disponível em: http://www.informationr.net/ir/9-3/paper175.html. Acesso em: 27 mar. 2ํ17.

SEALE, Clive. Quality in qualitative research. Quality Inquiry, v.5, n.4, p. 465-478, 1999. Disponível em: http://citeseerx.ist.psu.edu/viewdoc/download? doi=10.1.1.460.3511\&rep=rep1\&type=pdf. Acesso em: 10 ag. 2016. 
VIDICH, Arthur; LYMAN, Stanford. Qualitative methods: their history in sociology an anthropology. In: DENZIN, Norman and LINCOLN, Yvonna (Editors). Handbook of qualitative research. Thousand Oaks: Sage Publications, 1994, p.23-47.

\section{Recebido: 2017-08-25}

Aprovado: 2017-08-27

DOI: $10.3895 /$ rbect.v11n1.6977

Como citar: MOREIRA, H. Critérios e estratégias para garantir o rigor na pesquisa qualitativa. Revista Brasileira de Ensino de Ciência e Tecnologia, v. 11, n. 1, 2018. Disponível em: <https://periodicos.utfpr.edu.br/rbect/article/view/6977>. Acesso em: $\mathrm{xxx}$.

Correspondência: Herivelto Moreira - herivelto.moreira51@gmail.com Direito autoral: Este artigo está licenciado sob os termos da Licença Creative Commons-Atribuição 4.0 Internacional.

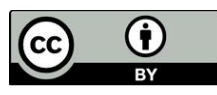

